\title{
CLEOPATRA sheds light on how to tackle metastatic disease
}

Metastatic breast cancer that overexpresses HER2 has historically been one of the most aggressive forms of the disease, and is associated with a poor prognosis. The comprehensive HER2 blockade achieved with the combination of the anti-HER2 humanized monoclonal antibodies pertuzumab and trastuzumab plus chemotherapy has resulted in a significant improvement in overall survival and progressionfree survival (PFS) for patients with this disease; these beneficial results have now been consolidated with the long term follow-up results of the CLEOPATRA study conducted in 204 centres across 25 countries by Sandra M. Swain and colleagues.

In this double-blind phase III trial, patients with HER2positive metastatic breast cancer who had not received previous chemotherapy or biological treatment for metastatic disease were randomly assigned to receive either pertuzumab, trastuzumab and docetaxel ( $n=402)$, or placebo, trastuzumab and docetaxel $(n=406)$. The longest duration of follow up was approximately 6 years. In a very conservative analysis, "overall survival was analysed using all randomized patients, with no adjustments for crossover once the study treatments were unblinded," explains Swain. She continues, "patients who crossed over from the placebo to the pertuzumab arm were analysed as part of the placebo arm".

At a median follow up of 50 months, the investigators reported an increase in median overall survival of 15.7 months in the pertuzumab arm compared with the placebo arm (56.5 months versus 40.8 months). "This survival improvement is unprecedented among studies of metastatic breast cancer" highlights Swain, "a result that

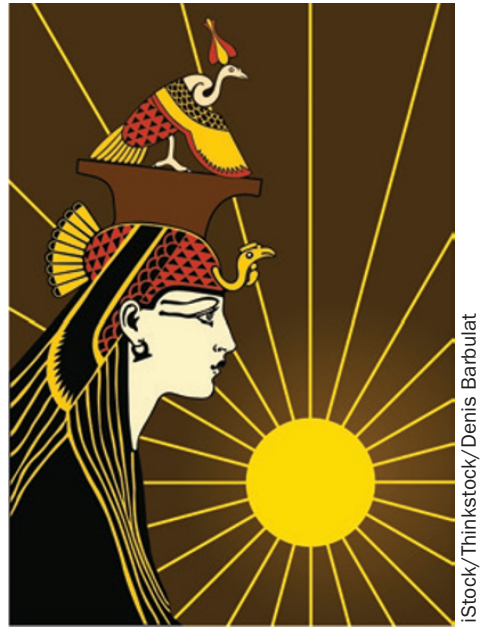

will be incredibly meaningful to patients and their families".

The increase in median PFS of 6.3 months in the pertuzumab group, reported in May 2012 at the interim analysis, was maintained after long-term follow up. Among patients who had a confirmed partial response or complete response at the time of primary analysis, those in the pertuzumab group had an 8-month longer duration of response than patients in the placebo group. Of note, the safety profile that included the long-term cardiac safety of the pertuzumab regimen, was unchanged from previous analysis.

Despite these encouraging results, most of the deaths reported in the study were breast cancer related, underlining the need for better treatment options. In addition, these findings raise important questions on the use of pertuzumab in the management of patients with HER2-positive early stage breast cancer, which is being investigated in the ongoing APHINITY trial (NCT01358877) in nearly 5,000 patients with early stage disease.

\section{Alessia Errico}

Original article Swain, S. M. et al. Pertuzumab, trastuzumab, and docetaxel in HER2-positive metastatic breast cancer. N. Engl. J. Med. doi:10.1056/NEJMoa1413513 